Research Article

\title{
Some New Variants of Relative Regularity via Regularly Closed Sets
}

\author{
Sehar Shakeel Raina $(\mathbb{D})$ and A. K. Das $\mathbb{1}$ \\ School of Mathematics, Shri Mata Vaishno Devi University, Katra 182320, Jammu and Kashmir, India \\ Correspondence should be addressed to A. K. Das; akdasdu@yahoo.co.in
}

Received 20 April 2021; Accepted 6 May 2021; Published 25 May 2021

Academic Editor: Frédéric Mynard

Copyright ( $) 2021$ Sehar Shakeel Raina and A. K. Das. This is an open access article distributed under the Creative Commons Attribution License, which permits unrestricted use, distribution, and reproduction in any medium, provided the original work is properly cited.

\begin{abstract}
Every topological property can be associated with its relative version in such a way that when smaller space coincides with larger space, then this relative property coincides with the absolute one. This notion of relative topological properties was introduced by Arhangel'skii and Ganedi in 1989. Singal and Arya introduced the concepts of almost regular spaces in 1969 and almost completely regular spaces in 1970. In this paper, we have studied various relative versions of almost regularity, complete regularity, and almost complete regularity. We investigated some of their properties and established relationships of these spaces with each other and with the existing relative properties.
\end{abstract}

\section{Introduction}

Arhangel'skii and Ganedi in [1] introduced the concept that with each topological property $\mathscr{P}$ one can associate a relative version of it formulated in terms of location of $Y$ in $X$ in such a natural way that when $Y$ coincides with $X$, then this relative property coincides with $\mathscr{P}$. The motivation behind this study is to investiagte how the smaller space is located in the larger one. In past few years, a lot of work has been done on relative topology. Grothendieck [2], Tkachuk [3], Chigogidze [4], Ranchin [5], Dow and Varmeer [6], Das and Bhat [7], Das and Raina [8], Just and Tartir [9], and so on are few names who remarkably contributed in this area. An important feature of relative topology is that a whole pack of relative topological properties can be relativised from a single absolute topological property. It depends on the absolute property that how many interesting versions of relative properties can be created out of it.

In general topology, regularity and normality play a very prominent role in studying topological properties which were widely studied by researchers. Like normality, relative version of regularity has also been studied by Arhangel'skii in [10]. In a survey article, Arhangel'skii also studied some versions of relative regularity like relative strong regular, relative superregular, and so on. There are still many important versions of regularity studied by different topologists in general topology which need to be studied in relative sense. Complete regularity is an important classical version of regularity in which a point and a closed set can be functionally separated. Almost regularity and almost complete regularity are some other versions of regularity introduced by Singal and Arya in $[11,12]$. In this paper, we have studied these concepts in relative sense. We have also defined relative almost strongly regular and relative almost superregular spaces. Relationships of these notions with each other and with the existing notions have also been studied and counterexamples are provided wherever needed. It is observed that relative almost regularity is the most generalized among all the versions of relative regularity.

\section{Basic Definitions and Preliminaries}

Let $X$ be a topological space and let $A \subset X$. A set $U \subset X$ is said to be regularly open [13] if $U=\bar{U}^{o}$. The complement of a regularly open set is called regularly closed. A topological space $X$ is said to be almost normal [11] if for every pair of disjoint sets $A$ and $B$ one of which is regularly closed and other is closed, there exist disjoint open sets $U$ and $V$ such that $A \subset U$ and $B \subset V$. A topological space $X$ is said to be completely regular if for every closed set $A$ of $X$ and every 
point $x \in X$ such that $x \notin A$, there exists a continuous function $f$ on $X$ into the closed interval $[0,1]$ such that $f(x)=0$ and $f(A)=1$. A topological space $X$ is said to be almost regular [12] if for every regularly closed set $A$ and a point $x \notin A$, there exist disjoint open sets $U$ and $V$ such that $x \in U$ and $A \subset V$. A topological space $X$ is said to be almost completely regular [11] if for every regularly closed set $A$ of $X$ and a point $x \in X$ such that $x \notin A$, there exists a continuous function $f$ on $X$ into the closed interval $[0,1]$ such that $f(x)=0$ and $f(A)=1$.

Let $Y \subset X . Y$ is said to be $T_{1}$ in $X$ or relative $T_{1}$ [10] if for every $y \in Y,\{y\}$ is closed in $X$. $Y$ is said to be Hausdorff in $X$ or relative Hausdorff [10] if for every pair of distinct points in $Y$ there exist disjoint open sets separating them. $Y$ is said to be regular in $X$ [10] or relative regular if for every closed set $A$ of $X$ and a point $y \in Y$ such that $y \notin A$ there exist disjoint open subsets $U$ and $V$ of $X$ such that $A \cap Y \subset U$ and $y \in V$. $Y$ is said to be normal in $X$ or relative normal [10] if for every pair of disjoint closed sets $A$ and $B$ of $X$, there exist disjoint open subsets $U$ and $V$ of $X$ such that $A \cap Y \subset U$ and $B \cap Y \subset V$.

\section{Relative Almost Regular Spaces}

Definition 1. Let $X$ be a topological space and $Y \subset X . Y$ is said to be almost regular in $X$ or relative almost regular (almost superregular in $X$ or relative almost superregular) if for every regularly closed set $A$ in $X$ and a point $y \in Y$ such that $y \notin A$, there exist disjoint open sets $U$ and $V$ in $X$ such that $A \cap Y \subset U$ and $y \in V(A \subset U$ and $y \in V)$.

Definition 2. Let $X$ be a topological space and $Y \subset X . Y$ is said to be almost strongly regular in $X$ or relative almost strongly regular if for every regularly closed set $A$ in $X$ and a point $x \in X$ such that $x \notin A$, there exist disjoint open sets $U$ and $V$ in $X$ such that $A \cap Y \subset U$ and $x \in V$.

Clearly, if $X$ is almost regular, then $Y$ is almost regular in $X$. If $Y$ is regular in $X$, then $Y$ is almost regular in $X$. If $Y$ is almost superregular in $X$ (almost strongly regular in $X$ ), then $Y$ is almost regular in $X$. But none of these implications can be reversed. Also, $Y$ can be almost superregular in $X$ without being almost strongly regular in $X$, and vice versa, $Y$ can be almost strongly regular in $X$ without being almost superregular in $X$.

Example 1. Let $X=\mathbb{R}$. Define a topology on $X$ by declaring any set open if the set is either $X$ or excludes 0 . This space is called excluded point topology with 0 as excluded point. Let $Y=\mathbb{R} \backslash\{0\}$. Here $X$ is not almost regular and $Y$ is not relative almost superregular because every regularly closed set in $X$ includes 0 and there does not exist any open set containing 0 . But $Y$ is relative almost strongly regular in $X$ as for every regularly closed set $A$ in $X$ and a point $x \in X$ such that $x \notin A$ there exist disjoint open sets $U$ and $V$ in $X$ such that $A \cap Y \subset U$ and $x \in V$.

Example 2. Let $X$ be the set of all integers. Define a topology $\tau$ on $X$, where every odd integer is open and a set $U$ is open for every even integer $p \in U$, and the successor and the predecessor of $p$ also belong to $U$. Let $Y=\{2,6,10,14, \ldots, 2(2 n+1), \ldots\}$, where $n=0,1,2, \ldots, Y$ is not almost strongly regular in $X$ because $\{2,3,4\}$ is regularly closed in $X, 1 \in X$, and there do not exist disjoint open sets in $X$ separating $\{2,3,4\} \cap Y=\{2\}$ and 1 . But $Y$ is almost regular in $X$.

Example 3. Let $X=\{a, b, c\}$ and $T=\{\{a\},\{b\},\{a, b\}, X, \phi\}$ be a topology on $X$. Let $Y=\{c\}$. $X$ is not almost normal and $Y$ is not almost strongly regular in $X$ because $\{b, c\}$ is regularly closed in $X$ and for $\{b, c\} \cap Y=\{c\}$ and $a \in X$, there do not exist disjoint open sets in $X$ separating them. But $Y$ is vacuously almost superregular in $X$ as $\{a, c\},\{b, c\}$ are the only regularly closed sets in $X$ and there does not exist any $y \in Y$ which does not belong to these sets.

Arhangel'skii in [10] defined relative superregular and relative strongly regular as follows: $Y \subset X$ is said to be superregular in $X$ if for every closed set $A$ in $X$ and a point $y \in Y$ such that $y \notin A$, there exist disjoint open sets $U$ and $V$ in $X$ such that $A \subset U$ and $y \in V$. And $Y$ is said to be strongly regular in $X$ if for every closed set $A$ in $X$ and a point $x \in X$ such that $x \notin A$, there exist disjoint open sets $U$ and $V$ in $X$ such that $A \cap Y \subset U$ and $x \in V$. The idea of defining relative almost superregular and relative almost strongly regular is taken from these concepts. It is clear from the definitions that relative strongly regular implies relative almost strongly regular and relative superregular implies relative almost superregular. But from the following example, it can be easily checked that none can be reversed.

Example 4. Let $X=\{a, b, c\}$ and $\tau=\{\{b\},\{a, b\},\{b, c\}, X, \phi\}$ be a topology on $X$. Let $Y=\{b, c\}$. Since there does not exist any regularly closed set in $X, Y$ is vacuously relative almost superregular and relative almost strongly regular in $X$. But $Y$ is neither relative superregular nor relative strongly regular because for the closed set $\{c\}$ in $X$ and $b \in X$, there do not exist disjoint open sets in $X$ satisfying the conditions of relative superregularity or relative strong regularity.

Theorem 1. Let $Y \subset X$. Then, the following statements are equivalent.

(a) $Y$ is relative almost superregular.

(b) For each $y \in Y$ and for each regularly open set $V$ of $X$ containing $y$, there exists regularly open set $U$ in $X$ such that $y \in U \subset \bar{U} \subset V$.

(c) For every regularly closed set $A$ of $X$ and each $y \in Y$ such that $y \notin A$, there exist open sets $U$ and $V$ in $X$ such that $y \in U, A \subset V$, and $\bar{U} \cap \bar{V}=\phi$.

Proof

$(a) \Rightarrow(b)$ Let $y \in Y$ and $V$ be a regularly open set in $X$ containing $y$. Then, $X \backslash V$ is a regularly closed set in $X$ and $y \notin X \backslash V$. Since $Y$ is relative almost superregular, there exist disjoint open sets $U_{1}$ and $U_{2}$ in $X$ such that $y \in U_{1}$ and $X \backslash V \subset U_{2}$. Since $U_{1} \cup U_{2}=\phi$, then $\overline{U_{1}} \cup U_{2}=\phi$, and hence $\overline{U_{1}} \subset X \backslash U_{2} \subset V$. Thus, 
$y \in U_{1} \subset \overline{U_{1}} \subset V$. Now $U_{1} \subset \overline{U_{1}} \subset \overline{U_{1}} \subset V$. Take ${\overline{U_{1}}}^{\circ}=U$. Then, $U$ is a regularly open set in $X$ and $y \in U \subset \bar{U} \subset V$.

$(b) \Rightarrow(c)$ Let $A$ be a regularly closed set in $X$ and $y \in Y$ such that $y \notin A$. Since $X \backslash A$ is regularly open set in $X$ containing $y$, there exists regularly open set $V$ in $X$ such that $y \in V \subset \bar{V} \subset X \backslash A$. Again since $V$ is a regularly open set in $X$ containing $y$, there exists a regularly open set $U$ in $X$ such that $y \in U \subset \bar{U} \subset V$. Now $U$ and $X \backslash \bar{V}$ are two open sets in $X$ with disjoint closures containing $y$ and $A$, respectively.

$(c) \Rightarrow(a)$ Let $A$ be a regularly closed set in $X$ and $y \in Y$ such that $y \notin A$. Then, there exist open sets $U$ and $V$ in $X$ with disjoint closures containing $y$ and $A$, respectively. Thus, $U$ and $X \backslash \bar{U}$ are disjoint open sets in $X$ containing $y$ and $A$, respectively. Hence, $Y$ is relative almost superregular.

Theorem 2. Let $Y \subset X$. Then, for each $y \in Y$ and for each regularly open set $V$ of $X$ containing $y$, there exists regularly open set $U$ such that $y \in U \subset \bar{U} \subset V$ implies that for each $y \in Y$ and for each neighborhood $M$ of $y$ in $X$, there exists regularly open neighborhood $V$ of $y$ in $X$ such that $\bar{V} \subset \overline{M^{\circ}}$.

Proof. Let $y \in Y$ and $M$ be a neighborhood of $y$ in $X$. Then, there exists an open set $U$ in $X$ such that $y \in U \subset M$ implies $y \in U \subset \overline{U^{o}} \subset \bar{U} \subset \overline{M^{o}}$. $U$ is open implies that $\bar{U}^{o}$ is regularly open in $X$ containing $y$ and so there exists a regularly open set $V$ containing $y$ such that $y \in V \subset \bar{V} \subset \bar{U}^{O} \subset \bar{U} \subset \overline{M^{o}}$. Thus, $y \in V \subset \bar{V} \subset \overline{M^{\circ}}$. 1.

The proof of the following result is similar to Theorem

Theorem 3. Let $Y \subset X$. Then, the following statements are equivalent.

(a) $Y$ is relative superregular.

(b) For each $y \in Y$ and for each open set $V$ of $X$ containing $y$, there exists open set $U$ in $X$ such that $y \in U \subset \bar{U} \subset V$.

(c) For every closed set $A$ of $X$ and each $y \in Y$ such that $y \notin A$, there exist open sets $U$ and $V$ in $X$ such that $y \in U, A \subset V$ and $\bar{U} \cap \bar{V}=\phi$.

Definition 3 (see [14]). A space is semiregular if the regularly open sets form a base for the topology.

Theorem 4 (see [12]). A space $X$ is semiregular if for every $x \in X$ and for each open set $V$ containing $x$, there exists an open set $U$ such that $x \in U \subset \overline{U^{o}} \subset V$.

Theorem 5. In a semiregular space, every relative almost superregular space is relative superregular.

Proof. Let $X$ be a semiregular space and $Y \subset X$. Let $y \in Y$ and $V$ be any set containing $y$. Since $X$ is semiregular, there exists an open set $U$ in $X$ such that $y \in U \subset \overline{U^{o}} \subset V$. Again since $U$ is open neighborhood of $y$ in $X$ and $Y$ is relative almost superregular, there exists an open set $W$ such that $y \in W \subset \bar{W} \subset \overline{U^{o}}$, which implies $y \in W \subset \bar{W} \subset V$. Hence, $Y$ is relative superregular.

Corollary 1. $Y \subset X$ is relative superregular iff $Y$ is relative almost superregular and $X$ is semiregular space.

Recall that a space $X$ is said to be a Urysohn space if for every pair of distinct points on $X$, there exists two open sets in $X$ with disjoint closures separating them, respectively.

Theorem 6. Every relative almost superregular, relative Hausdorff space is a Urysohn space.

Proof. Let $Y \subset X$ be a relative almost superregular, relative Hausdorff space. Let $y_{1}$ and $y_{2}$ be two distinct points in $Y$. Since $Y$ is relative Hausdorff, there exist $U$ and $V$ disjoint open sets in $X$ such that $y_{1} \in U$ and $y_{2} \in V$. $U \cap V=\phi$ implies $\bar{U} \cap V=\phi$. Since $X \backslash \bar{U}$ is a regularly open set in $X$ containing $y_{2}$ and $Y$ is relative almost superregular, there exists an open set $W$ in $X$ such that $y_{2} \in W \subset \bar{W} \subset X \backslash \bar{U}$. This implies that $y_{1} \in U$ and $y_{2} \in W$ and $\bar{U} \cap \bar{W}=\phi$.

Theorem 7. If $Y \subset X$ is relative almost regular, then for each point $y \in Y$ and each regularly open set $V$ containing $y$, there exists a regularly open set $U$ in $X$ such that $y \in U \subset \bar{U} \subset V \cup X \backslash Y$.

Proof. Let $Y \subset X$ be relative almost regular. Let $y \in Y$ and $V$ be a regularly open set in $X$. Then, $X \backslash V$ is regularly closed in $X$ such that $y \notin X \backslash V$. By relative almost regularity of $Y$, there exist disjoint open sets $U_{1}$ and $U_{2}$ in $X$ such that $y \in U_{1}$ and $(X \backslash V) \cap Y \subset U_{2}$. Now $U_{1} \cap U_{2}=\phi$ implies that $\overline{U_{1}} \cap U_{2}=\phi$, which implies $\overline{U_{1}} \subset X \backslash Y$. Thus, $y \in U_{1} \subset \overline{U_{1}} \subset V \cup X \backslash Y$.

Example 5. Let $X=\{a, b, c, d\}$ and $T_{X}=\{\{d\},\{b, d\},\{c, d\}$, $\{b, c, d\}, X, \phi\}$ be a topology on $X$. Let $Y=\{a, b, c\}$. $T_{Y}=\{\{b\},\{c\},\{b, c\}, Y, \phi\}$. Clearly, $Y$ is closed and relative almost regular in $X$. But $Y$ is not almost regular in itself that is with respect to the subspace topology $T_{Y}$ because $\{a, c\}$ is regularly closed in $Y$ and $b \in Y$ cannot be separated by disjoint open sets in $Y$.

Remark 1. From the above example, closed relative almost regular space is not almost regular, i.e., if $Y$ is closed in $X$ and $Y$ is relative almost regular in $X$, then $Y$ is not necessarily almost regular in itself. Thus, the following result is relevant.

Theorem 8. Every closed, relative almost regular, relative Hausdorff space is a Urysohn space.

Proof. Let $Y \subset X$ be closed and relative regular, relative Hausdorff space. Let $y_{1}$ and $y_{2}$ be two distinct points in $Y$. Since $Y$ is relative Hausdorff, there exist disjoint open sets $U$ and $V$ in $X$ such that $y_{1} \in U$ and $y_{2} \in V . U \cap V=\phi$ implies $\bar{U} \cap V=\phi$. Since $X \backslash \bar{U}$ is a regularly closed set in $X$ containing $y_{2}$ and $Y$ is relative almost regular, there exists an open set $W$ in $X$ such that $y_{2} \in W \subset \bar{W} \subset X \backslash \bar{U} \cup X \backslash Y$ 
which implies $y_{2} \in W \subset \bar{W} \subset X \backslash(\bar{U} \cup Y)$. Thus, $y_{2} \in W \cap Y, y_{1} \in U \cap Y$ and $\bar{U} \cap(\bar{W} \cap Y)=\phi$. Using the fact that $Y$ is closed, it can be easily shown that $\overline{U \cap Y} \cap \overline{W \cap Y}=\phi$. Hence, $Y$ is a Urysohn space.

In above result, the closedness of $Y$ cannot be dropped (see the following example).

Example 6. Consider Example 3. Let $Y=\{a, b\}$. Then, $Y$ is not closed but relative Hausdorff and relative almost regular. But it can be easily checked that $Y$ is not a Urysohn space.

Definition 4 (see [15]). $Y \subset X$ is said to be almost normal in $X$ or relative almost normal if for every pair of disjoint closed sets $A$ and $B$ of $X$, such that $A$ is regularly closed there exist disjoint open subsets $U$ and $V$ of $X$ such that $A \cap Y \subset U$ and $B \cap Y \subset V$.

Theorem 9. If $Y$ is relative almost normal and relative $T_{1}$, then $Y$ is relative almost regular.

Proof. Let $A$ be a regularly closed set in $X$ and $y \notin A$ be a point in $Y$. Since $Y$ is $T_{1},\{y\}$ is closed in $X$. Since $Y$ is almost normal in $X$, there exist disjoint open sets $U$ and $V$ such that $A \cap Y \subset U$ and $\{y\} \subset V$. Hence, $Y$ is almost regular in $X$.

The following example establishes that relative almost normality does not necessarily imply relative almost regularity.

Example 7. Let $X=\mathbb{R}$ with same topology as in Example 1. Let $Y=A \cup\{0\}$, where $A$ is any finite subset of $X$. $Y$ is not relative almost regular because for $\{0,1\}$, which is regularly closed in $X$, and $p$ any point in $A$ other than 1 , there does not exist disjoint open sets separating them. But $Y$ is vacuously relative almost normal as there does not exist any pair of disjoint regularly closed sets in $X$.

Definition 5 (see $[16,17]) . X$ is said to be almost $\beta$-normal if for any two disjoint closed subsets $A$ and $B$ of $X$ such that $A$ is regularly closed, there exist open subsets $U$ and $V$ of $X$ such that $A \cap U$ is dense in $A$ and $B \cap V$ is dense in $B$ and $\bar{U} \cap \bar{V}=\phi$.

Theorem 10. In an almost $\beta$-normal space, every relative $T_{1}$ space is relative almost superregular.

Proof. Let $A$ be a regularly closed set in $X$ and $y \in Y$ such that $y \notin A$. Since $Y$ is relative $T_{1},\{y\}$ is closed in $X$. Now since $X$ is almost $\beta$-normal, there exist disjoint open sets $U$ and $V$ in $X$ such that $\overline{A \cap U}=A$ and $y \in V$. Since $A \subset \bar{U}, V$ and $X \backslash \bar{V}$ are disjoint open sets in $X$ containing $y$ and $A$, respectively. Hence, $Y$ is almost superregular in $X$.

In the above theorem, the condition of relative $T_{1}$ cannot be relaxed as is shown in the following example.

Example 8. Consider the space $X$ in Example 1. $X$ is vacuously normal and thus almost $\beta$-normal. $Y$ is not relative $T_{1}$ because none of the singletons of $Y$ is closed in $X$. Also, $Y$ is not relative almost superregular.
Remark 2. From the above example, we have also observed that relative Hausdorff does not imply relative $T_{1}$. But another relative version of $T_{1}$ space can be defined which satisfies the condition that for every pair of distinct points $y_{1}$ and $y_{2}$ in $Y$, there exist disjoint open sets $U$ and $V$ in $X$ such that $y_{1} \in U, y_{2} \notin U$ and $y_{2} \in V, y_{1} \notin V$. In that case, relative Hausdorff implies this relative version of $T_{1}$ space.

Definition 6. A space $X$ is said to be seminormal if for every closed set $A$ contained in an open set $U$, there exists a regularly open set $V$ such that $A \subset V \subset U$.

Theorem 11. Every relative $T_{1}$ subset of a seminormal, almost $\beta$-normal space is relative superregular.

Proof. Let $A$ be a closed set in $X$ and $y \in Y$ such that $y \notin A$. Since $Y$ is relative $T_{1},\{y\}$ is closed in $X$. By seminormality of $X$, there exists regularly open set $U$ such that $\{y\} \subset U \subset X \backslash A$. Here $F=X \backslash U$ is a regularly closed set containing $A$ and $y \notin F$. Now since $X$ is almost $\beta$-normal and $Y$ is relative $T_{1}$, by Theorem $10, Y$ is relative almost superregular. Thus, there exist disjoint open sets $V$ and $W$ in $X$ such that $y \in V$ and $A \subset F \subset W$. Hence, $Y$ is relative superregular.

Continuous image of a relative almost superregular (relative almost strongly regular) space is not relative almost superregular (relative almost strongly regular) space. Following is an example.

\section{Example 9}

(1) Let $X=\{a, b, c, d\}$. Let $\tau_{1}$ be the discrete topology on $X$ and $\tau_{2}=\{\{a\},\{b, c\},\{a, b, c\}, X, \phi\}$ be a topology on $X$. Let $Y=\{a, b, c\}$. $Y$ is relative almost superregular and relative almost strongly regular. Define $f:\left(X, \tau_{1}\right) \longrightarrow\left(X, \tau_{2}\right)$ as $f(a)=a, \quad f(b)=d$, $f(c)=c$, and $f(d)=b$. Then, $f$ is continuous, oneone, and onto function. But $f(Y)=\{a, c, d\}$ is not even relative almost regular in $\left(X, \tau_{2}\right)$ because $A=$ $\{a, d\}$ is regularly closed in $\left(X, \tau_{2}\right)$ and $c \in Y, c \notin A$ cannot be separated by disjoint open sets in $\left(X, \tau_{2}\right)$.

(2) Also, take $X=\mathbb{R}$ with $\tau_{1}$ as discrete space and $\tau_{2}$ as excluded point topology with 0 as excluded point. Let $Y$ be the same as Example 7 which is relative almost superregular and relative almost strongly regular in $\tau_{1}$. Define $f:\left(X, \tau_{1}\right) \longrightarrow\left(X, \tau_{2}\right)$ as identity map which is continuous, one-one, and onto. But Example 7 shows that $f(Y)$ is not even relative almost regular in $\tau_{2}$.

In ([16], Theorem 2.25), it is observed that inverse image of a regularly closed set under an onto, continuous, open, and closed mapping is regularly closed.

Theorem 12. The image of a relative almost regular space is invariant under an onto, continuous, open, and closed mapping. 
Proof. Let $X_{1}$ and $X_{2}$ be two topological spaces, $Y$ is relative almost regular in $X_{1}$, and $f: X \longrightarrow Y$ is onto, continuous, open, and closed mapping. Let $A$ be a regularly closed set in $X_{2}$ and $y \in Y$ such that $y \notin A$. Then, $f^{-1}(A)$ is regularly closed in $X_{1}, f^{-1}(y) \in Y$, and $f^{-1}(y) \notin f^{-1}(A)$. Since $Y$ is relative almost regular in $X_{1}$, there exist disjoint open sets $U$ and $V$ in $X_{1}$ such that $f^{-1}(A) \cap Y \subset U$ and $f^{-1}(y) \in V$. Since $U \cap V=\phi, \quad f^{-1}(A) \cap Y \cap \bar{V}=\phi \quad$ which implies $A \cap f(Y) \cap f(\bar{V})=\phi$. Also, $\bar{V}$ is closed implies that $f(\bar{V})$ is closed. Since $f(\bar{V})$ is a closed set containing $f(V)$, $\overline{f(V)} \subset f(\bar{V})$. So, $\overline{f(V)} \cap A \cap f(Y)=\phi$. Thus, there are two disjoint open sets $X_{2} \backslash \overline{f(V)}$ and $f(V)$ in $X_{2}$ such that $A \cap f(Y) \subset X_{2} \backslash \overline{f(V)}$ and $y \in f(V)$. Hence, $f(Y)$ is relative almost regular in $X_{2}$.

\section{Relative Almost Completely Regular Spaces}

Definition 7. Let $X$ be a topological space and $Y \subset X$. Then, $Y$ is said to be completely regular in $X$ if for every closed set $A$ of $X$ and a point $y \in Y$ such that $y \notin A$, there exists a continuous function $f$ on $X$ into the closed interval $[0,1]$ such that $f(y)=0$ and $f(A \cap Y)=1$.

Definition 8. Let $X$ be a topological space and $Y \subset X$. Then, $Y$ is said to be almost completely regular in $X$ if for every regularly closed set $A$ of $X$ and a point $y \in Y$ such that $y \notin A$, there exists a continuous function $f$ on $X$ into the closed interval $[0,1]$ such that $f(y)=0$ and $f(A \cap Y)=1$.

Theorem 13. If $Y$ is completely regular in $X$, then $Y$ is almost completely regular in $X$.

Proof. This proof follows from the fact that every regularly closed set is closed.

Theorem 14. If $Y$ is almost completely regular in $X$, then $Y$ is almost regular in $X$.

Proof. Suppose $Y$ is almost completely regular in $X$. To prove that $Y$ is almost regular in $X$, let $A$ be a regularly closed set in $X$ and $y \in Y$ such that $y \notin A$. Since $Y$ is almost completely regular in $X$, there exists a continuous function $f: X \longrightarrow[0,1]$ such that $f(y)=0$ and $f(A \cap Y)=1$, which implies that $f^{-1}(1 / 2,1]$ and $f^{-1}[0,1 / 2)$ are two disjoint open sets in $X$ such that $A \cap Y \subset f^{-1}(1 / 2,1]$ and $y \subset f^{-1}[0,1 / 2)$. Hence, $Y$ is almost regular in $X$.

Theorem 15. If $Y$ is completely regular in $X$, then $Y$ is regular in $X$.

Proof. Suppose $Y$ is completely regular in $X$. To prove that $Y$ is regular in $X$, let $A$ be a closed set in $X$ and $y \in Y$ such that $y \notin A$. Since $Y$ is completely regular in $X$, there exists a continuous function $f: X \longrightarrow[0,1]$ such that $f(y)=0$ and $f(A \cap Y)=1$, which implies that $f^{-1}(1 / 2,1]$ and $f^{-1}[0,1 / 2)$ are two disjoint open sets in $X$ such that $A \cap Y \subset f^{-1}(1 / 2,1]$ and $y \subset f^{-1}[0,1 / 2)$. Hence, $Y$ is regular in $X$.

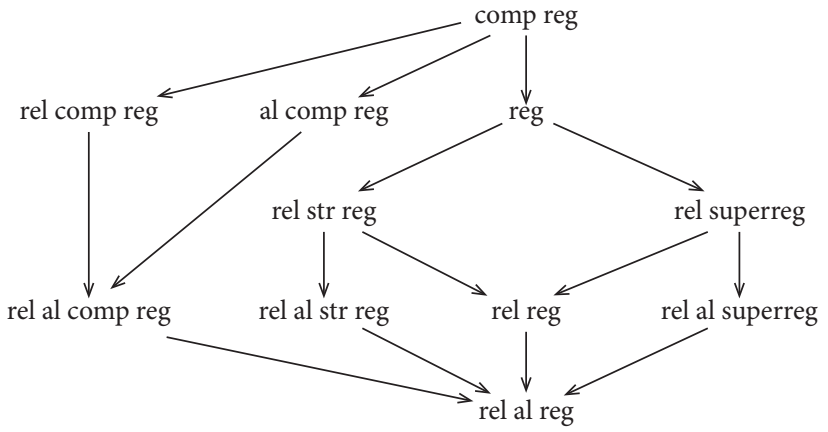

comp: completely superreg: superregular str: strongly

rel: relative reg: regular al: almost

Figure 1: Interrelation diagram.

Theorem 16 (see [15]). If $Y$ is relative almost normal, then for every pair of disjoint regularly closed sets $A$ and $B$ of $X$, there exists a continuous function $f$ on $X$ into closed interval $[0,1]$ such that $f(A \cap Y)=\{0\}$ and $f(A \cap Y)=\{1\}$.

Theorem 17. In a regular space, every relative almost normal space is relative completely regular.

Proof. Let $X$ be a regular space and $Y \subset X$ be relative almost normal. Let $A$ be a closed set in $X$ and $y \in Y$ such that $y \notin A$. Since $X$ is regular, there exist open sets $U$ and $V$ in $X$ such that $y \in U, A \cap Y \subset V$, and $\bar{U} \cap \bar{V}=\phi$. Now since $\bar{U}$ is regularly closed in $X, \bar{V}$ is closed in $X$, and $Y$ is relative almost normal, there exists continuous function $f: X \longrightarrow[0,1]$ such that $f(\bar{U} \cap Y)=0$ and $f(\bar{V} \cap Y)=1$. Thus, $f(y)=0$ and $f(A \cap Y)=1$. Hence, $Y$ is relative completely regular.

Theorem 18. In an almost regular space, every relative almost normal space is relative almost completely regular.

Proof. Let $A$ be a regularly closed set in $X$ and $y \in Y$ such that $y \notin A$. Since $X$ is almost regular, there exist open sets $U$ and $V$ in $X$ such that $y \in U, A \subset V$ and $\bar{U} \cap \bar{V}=\phi$. Rest of the proof is same as the proof of Theorem 17.

The proof of the following result is similar to the proof of the previous theorem.

Theorem 19. Every relative almost superregular, relative almost normal space is relative almost completely regular.

From Example 7, it is clear that in general, relative almost normal does not necessarily imply relative almost regular and hence not imply relative almost completely regular.

Theorem 20. In a normal space, every relative $T_{1}$ space is relative completely regular.

Proof. Let $A$ be a closed set in $X$ and $y \notin A$ be a point in $Y$. Since $Y$ is relative $T_{1},\{y\}$ is closed in $X$. By normality of $X$, there exists a continuous function $f: X \longrightarrow[0,1]$ such that 
$f(\{y\})=0$ and $f(A)=1$. Hence, $Y$ is relative completely regular.

Theorem 21. In an almost normal space, every relative $T_{1}$ space is relative almost completely regular.

Proof. Proof is similar to Theorem 20.

In above results, the condition of relative $T_{1}$ cannot be dropped. Consider Example 7 where $X$ has no disjoint closed sets, and hence $X$ is normal and almost normal as well. But, $Y$ is neither relative $T_{1}$ nor relative almost regular. Hence, $Y$ is neither relative completely regular nor relative almost completely regular.

From the definitions and implications shown in the paper, the interrelation in Figure 1 is obvious. Most of the relations are not reversible and it is natural to ask the following questions for further study on relative versions of variants discussed in the present paper.

\section{Question 1}

(1) Does there exist a relative almost regular space which is not relative almost completely regular?

(2) Does there exist a relative almost completely regular space which is not relative completely regular?

(3) Does there exist a relative almost completely regular space such that its larger space is not almost completely regular?

(4) Does there exist a relative completely regular space such that its larger space is not completely regular?

\section{Data Availability}

No data were used to support this study.

\section{Conflicts of Interest}

The authors declare that they have no conflicts of interest.

\section{Acknowledgments}

The first author is thankful to the Department of Science and Technology, Govt. of India, for awarding INSPIRE fellowship (IF160701).

\section{References}

[1] A. V. Arhangel'skii and K. M. M. Gennedi, Beginnings of the Theory of Relative Topological Properties, General Topology, Spaces and Mappings, MGU Moscow, Moscow, Russia, 1989.

[2] A. Grothendieck, "Crittres de compacitt dans les espaces fonctionnels gentraux," American Journal of Mathematics, vol. 74, pp. 175-185, 1952.

[3] V. V. Tkachuk, "On relative small inductive dimension," Vestnik Moskovskogo Universiteta, Seriya I. Matematika Mekhanika, vol. 5, pp. 22-25, 1982, in Russian.

[4] A. C. Chigogidze, "On relative dimensions," in General Topology, Spaces of Functions and DimensionMGU, Moscow, Russia, in Russian, 1985.
[5] D. V. Ranchin, "On compactness modulo an ideal," Doklady Akademii Nauk SSSR, vol. 202, pp. 761-764, 1972, in Russian.

[6] A. Dow and J. Vermeer, "An example concerning the property of a space being Lindelöf in another," Topology and Its Applications, vol. 51, no. 3, pp. 255-259, 1993.

[7] A. K. Das and P. Bhat, "A class of spaces containing all densely normal spaces," Indian Journal of Mathematics, vol. 57, no. 2, pp. 217-224, 2015.

[8] A. K. Das and S. S. Raina, "On relative $\beta$-normality," Acta Mathematica Hungarica, vol. 160, no. 2, pp. 468-477, 2020.

[9] W. Just and J. Tartir, "A \$ \$kappa \$-normal, not densely normal Tychonoff space," Proceedings of the American Mathematical Society, vol. 127, no. 3, pp. 901-905, 1999.

[10] A. V. Arhangel'skii, "A relative topological properties and relative topological spaces," Topology and its Applications, vol. 70, pp. 87-99, 1996.

[11] M. Singal and S. Arya, "Almost normal and almost completely regular spaces,” Kyungpook Mathematical Journal, vol. 25, no. 1, pp. 141-152, 1970.

[12] M. Singal and S. P. Arya, "On almost-regular spaces," Glasnik Matematicki Series III, vol. 4, no. 24, pp. 89-99, 1969.

[13] K. Kuratowski, Vol. I. (Translated from the French by J. Jawaroski) Academic Press, London and New York, PWN Polish Scientific Publishers. Warsaw, Warsaw, Cambridge University Press, Cambridge, UK, 1966.

[14] S. Willard, General Topology, Addison-Wesley pub. Co., Menlo Park CA, USA, 1970.

[15] S. S. Raina and A. K. Das, Some Variants of Normality in Relative Topological Spaces, Pre-Print.

[16] A. K. Das, P. Bhat, and J. K. Tartir, "On a simultaneous generalization of $\beta$-normality and almost normality," Filomat, vol. 31, no. 1, pp. 425-430, 2017.

[17] A. K. Das, P. Bhat, and J. K. Tartir, "Addendum to: on a simultaneous generalization of $\beta$-normality and almost normality," Filomat, vol. 31, no. 19, pp. 6021-6022, 2017. 